\title{
Basic research for the realization of online MEG using SSD
}

\author{
Kazuhiro Yagi \\ Interdisciplinary Graduate School of Agriculture and Engineering, University of Miyazaki, \\ 1-1, Gakuen Kibanadai-Nishi, Miyazaki, 889-2192, Japan \\ Yuta Shibahara \\ Graduate School of Engineering, University of Miyazaki, \\ 1-1, Gakuen Kibanadai-Nishi, Miyazaki, 889-2192, Japan \\ Lindsey Tate \\ Faculty of Engineering, University of Miyazaki, \\ 1-1, Gakuen Kibanadai-Nishi, Miyazaki, 889-2192, Japan \\ Keiko Sakurai \\ Faculty of Engineering, University of Miyazaki, \\ 1-1, Gakuen Kibanadai-Nishi, Miyazaki, 889-2192, Japan \\ Hiroki Tamura \\ Faculty of Engineering, University of Miyazaki, \\ 1-1, Gakuen Kibanadai-Nishi, Miyazaki, 889-2192, Japan
}

E-mail: kazuhiro_yagi@junwakai.com,hi16019@student.miyazaki-u.ac.jp,teitorinzeirini.c4@cc.miyazaki-u.ac.jp, sakurai.keiko.u6@cc.miyazaki-u.ac.jp,htamura@cc.miyazaki-u.ac.jp

\begin{abstract}
Neurofeedback systems have been found to be effective in the clinical rehabilitation of paralysis. However, most systems exist only for use with EEG, which is cumbersome to apply to patients and has lower spatial resolution than MEG. Furthermore, the best practices for neural data feature extraction and feature selection are not well established. The inclusion of the best performing feature extraction algorithms is critical to the development of clinical neurofeedback systems. Using simultaneously collected MEG and accelerometer data before and during 10 spontaneous finger movements, we performed an in-depth comparison of independent components analysis (ICA) and spatio-spectral decomposition (SSD) algorithms for their individual abilities to isolate movement-relevant features in brain activity. Having restricted raw data to that from sensorimotor rhythm (SMR) frequencies in select MEG sensors over sensorimotor cortex, we compared ICA and SSD components using: (1) 2D topographies, (2) activations over time, (3) and correlations with accelerometer data at both $0 \mathrm{~ms}$ and $60 \mathrm{~ms}$ time delays. SSD performed more quickly and produced components that were more highly correlated with the behavioral data than ICA. We will discuss these results and suggestions for application to neurofeedback systems. In particular, we will present detailed visualizations of SSD results and discuss potential strategies and pitfalls for feature selection.
\end{abstract}

Keywords: Magnetoencephalography, Spatio-spectral decomposition, Morlet wavelet transform, Neurofeedback

\section{Introduction}

For diseases that affect brain function, such as strokes, immediate treatment with medication and surgery is important, but post-onset rehabilitation also plays a critical role in the wellbeing of patients.

One of the techniques used for non-invasive brain function evaluation is magnetoencephalography $(\mathrm{MEG})^{1-}$

(C) The 2021 International Conference on Artificial Life and Robotics (ICAROB2021), January 21 to 24, 2021 
2. MEG has high temporal resolution as well as high spatial resolution, and it is commonly used clinically for epilepsy diagnosis and rehabilitation ${ }^{10}$. Without the ability to monitor a patient's relevant brain activity in real time during rehabilitative exercises, efficient rehabilitation cannot occur.

The purpose of this study was to evaluate the use of spatio-spectral decomposition (SSD) of real-time MEG data during spontaneous movement ${ }^{3-6}$. Performance was evaluated by comparing SSD results to the results from a standard analysis technique, independent components analysis (ICA). SSD completed decomposition faster than did ICA, with the SSD analysis completed about 270 times faster in the preliminary experiment. In addition, as shown in Table 1, the correlation with the accelerometer data (shifted by $60[\mathrm{~ms}]$ to account for the time difference between neural motor planning and actual motor execution) was also stronger for the most highly correlated SSD component as compared to the most highly correlated ICA component. Our results indicated that SSD outperforms ICA in the context of feature extraction for online, real-time MEG analysis; therefore, we present our investigation of the SSD components and conclude with suggestions for feature selection.

Table 1. The correlation with the accelerometer data and ICA or SSD.

\begin{tabular}{|c|c|}
\hline & $60[\mathrm{~ms}]$ \\
\hline 1st ICA & 0.71 \\
\hline 2nd ICA & -0.18 \\
\hline 3rd ICA & -0.55 \\
\hline 4th ICA & -0.07 \\
\hline 5th ICA & 0.35 \\
\hline 1st SSD & 0.15 \\
\hline 2nd SSD & -0.89 \\
\hline 3rd SSD & -0.13 \\
\hline 4th SSD & 0.47 \\
\hline 5th SSD & -0.45 \\
\hline
\end{tabular}

\section{Experiment}

The data were collected using a full-head 306-channel magnetoencephalograph (Vectorview, Elekta-Neuromag, Helsinki, Finland) at a sampling frequency of 1000 [Hz]. In order to reduce power supply noise and other interference, measurements were taken inside a magnetic field shield room $(1[\mathrm{kHz}]$ shielding rate $55.2[\mathrm{~dB}])$. The participant was a healthy person who attached an acceleration sensor to the middle finger of his left hand and performed ten spontaneous flexion (i.e., bending) and extension (i.e., relaxing) movements of the indicated finger as shown in Fig.1 (hereinafter referred to as "the task"). The accelerometer data indicated the start and duration of flexion and extension.
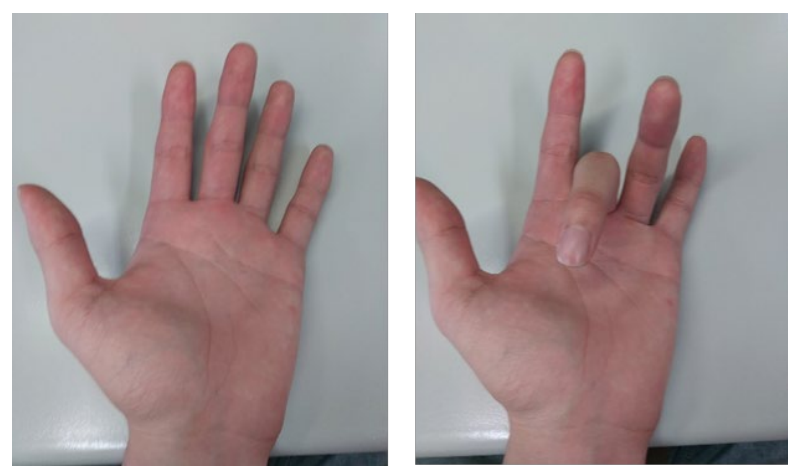

Fig.1: The spontaneous flexion (bending) and extension (relaxing) movements.

\section{Analysis Method}

After collecting the raw MEG data from all 306 channels, we sub-selected 26 gradiometers corresponding to the right sensorimotor cortex (SMC) and performed ICA and SSD analyses ${ }^{7-8}$, which each identified components within the largest cited frequency band for sensorimotor rhythm $(\mathrm{SMR})^{9}, 8-30[\mathrm{~Hz}]$. As presented in Table 1, we calculated Pearson correlations between the timeadjusted accelerometer data and each of the top five components from ICA and SSD. We calculated the SSD topographies about 20 seconds and then performed a Morlet wavelet transform to examine frequency power over time (Fig.2). In addition, in order to increase the signal-to-noise ratio (SNR), the wavelet analysis was performed on task-locked averaged components to indicate average brain activity in the final 2 seconds before flexion start. There were 10 tasks completed over 20 seconds at irregular intervals. 


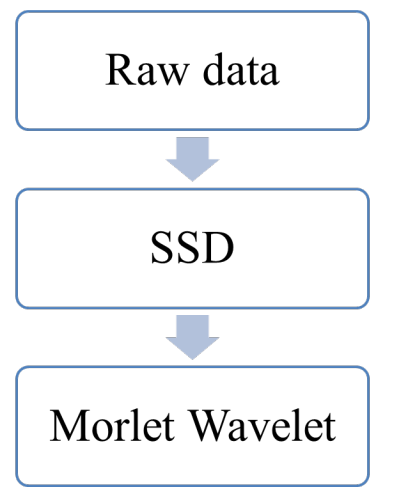

Fig.2: The flow of analysis method

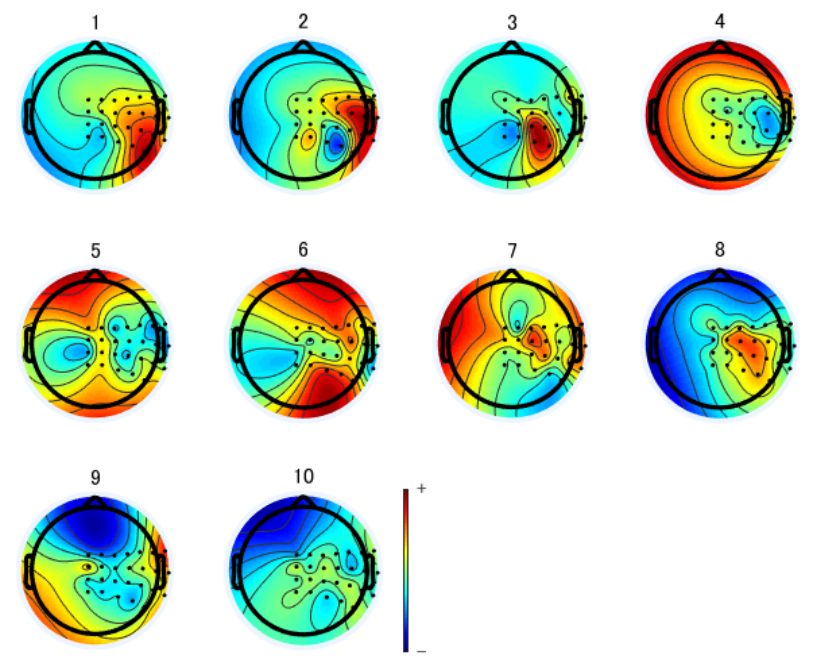

Fig.3: The results of Topography using SSD

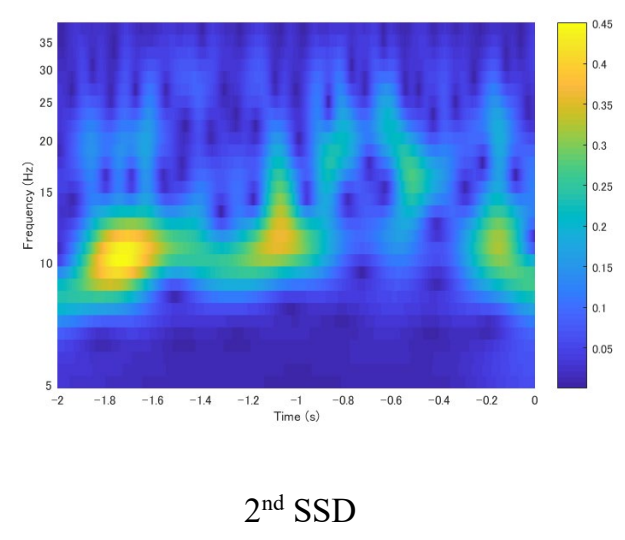

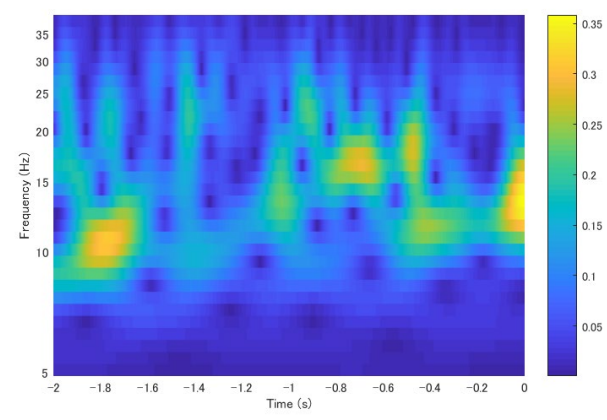

$3^{\text {rd }}$ SSD

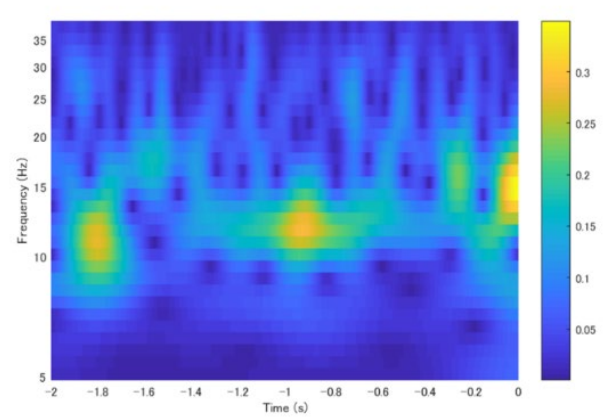

$4^{\text {th }} \mathrm{SSD}$

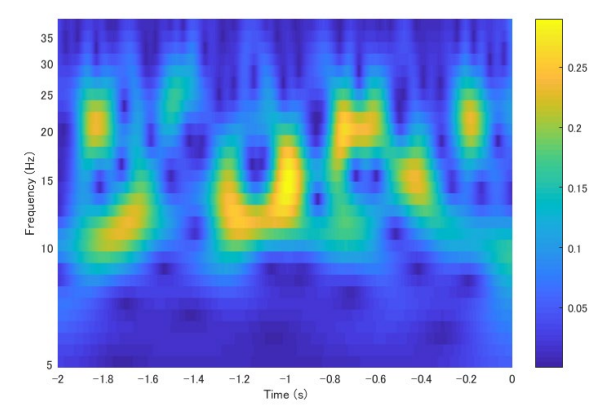

$5^{\text {th }} \mathrm{SSD}$

Fig.4: The results of the across-task averaged second, third, fourth and fifth SSD components

\section{Results}

From the results of topography (Fig. 3), the first eight SSD components showed activity localized around the right SMC. As shown in Fig. 4, the across-task averaged third and fourth SSD components had higher SMR band power immediately preceding the task. The pattern of activity observed in the SMR frequency band across task instances indicated that the first four SSD components

(C) The 2021 International Conference on Artificial Life and Robotics (ICAROB2021), January 21 to 24, 2021 
captured artifactual activity (e.g., movement activity). In the averaged component as well as across task instances, steady activity during the two seconds preceding flexion was observed at $12-13[\mathrm{~Hz}]$ in the fifth SSD component.

\section{Conclusion}

In this paper, we performed offline MEG data analysis during flexion and extension of the middle finger of the left hand in order to investigate the use of SSD in online, real-time MEG analysis.

There are several key points to take away regarding the automation of feature selection in this context. First, the SSD component with the highest correlation to accelerometer data (comp. 2, $r=-0.89$ ) was, in this case, indicative of muscular noise reaching the MEG sensors rather than indicating neural activity as desired. Second, the component most likely to indicate relevant neural activity (comp. 5) was, in addition to not being the most highly correlated with accelerometer data $(r=-0.45)$, also not the first component (i.e., it was not the component with the strongest eigenvalue or largest SNR). Finally, when only considering a few trials (i.e., at the beginning of neurofeedback rehabilitation) or particularly noisy data, using averaging techniques can lead to incorrect feature selection due to the extreme amplitude of artifacts and the components that capture them. Therefore, a component to use for patient feedback cannot be selected based solely on having the highest average SMR power, the highest correlation with behavioral data, or the highest eigenvalue.

Future study will include the same analysis for SSD components 6-8, which show promising topographies. Furthermore, we will investigate the usefulness of baseline correction in component selection and of logarithmically scaled components as features in unsupervised learning.

\section{References}

1. Franca Tecchio, Filippo Zappasodi, Mario Tombini, Antomio Oliviero, Patrizio Pasqualetti, Fabrizio Vernieri, Matilde Ercolani, Vittorio Pizzella, and Paolo Maria Rossini, Brain plasticity in recovery from stroke: An MEG assessment, NeuroImage 32 (2006) 1326 - 1334

2. Andrea Paggiaro, Niels Birbaumer, Marianna Cavinato, Cristina Turco, Emanuela Formaggio, Alessandra Del
Felice, Stefano Masiero and Francesco Piccione, Magnetoencephalography in Stroke Recovery and Rehabilitation, Frontiers in Neurology March 2016 Volume7 Article 35.

3. Vadim V. Nikulin, Guido Nolte, Gabriel Curio, A novel method for reliable and fast extraction of neuronal EEG/MEG oscillations on the basis of spatio-spectral decomposition, NeuroImage 55 (2011) 1528-1535.

4. Natalie Schaworonkow, Pedro Caldana Gordon, Paolo Belardinelli, Ulf Ziemann, Til Ole Bergmann and Christoph Zrenner, $\mu$-Rhythm Extracted With Personalized EEG Filters Correlates With Corticospinal Excitability in Real-Time Phase-Triggered EEG-TMS, Frontiers in Neuroscience December 2019 Volume12 Article954.

5. Stefan Haufe, Sven Dähne, Vadim V. Nikulin, Dimensionality reduction for the analysis of brain oscillations, NeuroImage 101 (2014) 583-597

6. Hanna-Leena Halme, Lauri Parkkonen, Comparing Features for Classification of MEG Responses to Motor Imagery, PLOS ONE December 16, 2016.

7. Franc,ois Tadel, 1 Sylvain Baillet, 2 John C.Mosher,3 Dimitrios Pantazis, 4 and Richard M. Leahy1, Brainstorm: A User-Friendly Application for MEG/EEG Analysis, Hindawi Publishing Corporation Computational Intelligence and Neuroscience Volume 2011, Article ID 879716, 13 pages.

8. Maren Stropahl, Anna-Katharina R. Bauer, Stefan Debener and Martin G. Bleichner, Source-Modeling Auditory Processes of EEG Data Using EEGLAB and Brainstorm, Frontiers in Neuroscience May 2018 Volume 12 Article 309.

9. Benjamin Blankertz, Claudia Sannelli, Sebastian Halder, Eva M. Hammer, Andrea Kübler, Klaus-Robert Müller, Gabriel Curio, Thorsten Dickhaus, Neurophysiological predictor of SMR-based BCI performance, NeuroImage 51 (2010) 1303-1309.

10. Stephen T. Foldes, Douglas J. Weber and Jennifer L. Collinger, MEG-based neurofeedback for hand rehabilitation, Journal of NeuroEngineering and Rehabilitation (2015) 12:85. 